\title{
THE RAMANUJAN IDENTITIES UNDER MODULAR SUBSTITUTIONS
}

\author{
BY \\ HANS RADEMACHER
}

1. Introduction. In connection with his discovery of certain divisibility properties of the partition function Ramanujan [1] $\left(^{1}\right)$ stated the identities

$$
\sum_{l=0}^{\infty} p(5 l+4) x^{l}=5 \frac{\Pi\left(1-x^{5 m}\right)^{5}}{\prod\left(1-x^{m}\right)^{6}}
$$

and

$$
\sum_{l=0}^{\infty} p(7 l+5) x^{l}=7 \frac{\prod\left(1-x^{7 m}\right)^{3}}{\Pi\left(1-x^{m}\right)^{4}}+49 x \frac{\Pi\left(1-x^{7 m}\right)^{7}}{\Pi\left(1-x^{m}\right)^{8}} .
$$

Here, as always in the sequel, the index $m$ in the infinite products runs through all positive integers. If these identities, for which various proofs have been given, are expressed in terms of the Dedekind $\eta$-function

$$
\eta(\tau)=e^{\pi i \tau / 12} \Pi\left(1-e^{2 \pi i m \tau}\right),
$$$$
\Im(\tau)>0,
$$

they appear in a form which suggests certain group-theoretical considerations, similar to those employed by Hecke in his theory of modular forms. In this way we transform the identities into new ones which are noteworthy because of the occurrence of the Legendre symbol and which, by a simple further argument, lead also to a proof of (1.1) and (1.2). An analogous identity for the modulus 13, given by Zuckerman, can be treated in the same way.

G. N. Watson and H. S. Zuckerman have also derived identities for the moduli $5^{2}$ and $7^{2}$. These will lead us to certain modular equations, which in turn will shed some light on those identities.

\section{Part I. Identities of Ramanujan and Zuckerman}

2. We have known since Euler

$$
\sum_{n=0}^{\infty} p(n) x^{n}=\frac{1}{\Pi\left(1-x^{m}\right)}
$$

with $p(0)=1$, or

$$
\frac{1}{\eta(\tau)}=e^{-\pi i \tau / 12} \sum_{n=0}^{\infty} p(n) e^{2 \pi i n \tau}
$$

Presented to the Society, February 22, 1941; received by the editors April 25, 1941.

(1) Numbers in square brackets refer to the bibliography at the end of this paper. 
Hence we obtain

$$
\begin{aligned}
\sum_{\lambda=0}^{4} \frac{1}{\eta\left(\frac{\tau+24 \lambda}{5}\right)} & =\sum_{\lambda=0}^{4} e^{-(\pi i \tau / 60)-(2 \pi i \lambda / 5)} \sum_{n=0}^{\infty} p(n) e^{(2 \pi i n \tau / 5)+(48 \pi i \lambda n / 5)} \\
& =e^{-\pi i \tau / 60} \sum_{n=0}^{\infty} p(n) e^{2 \pi i n \tau / 5} \sum_{\lambda=0}^{4} e^{-(2 \pi i / 5) \lambda(1-24 n)} \\
& =5 e^{-\pi i \tau / 60} \sum_{n \equiv 4(\bmod 5)} p(n) e^{2 \pi i n \tau / 5},
\end{aligned}
$$

and therefore

$$
\sum_{l=0}^{\infty} p(5 l+4) e^{2 \pi i l \tau}=\frac{1}{5} e^{-19 \pi i \tau / 12} \sum_{\lambda=0}^{4} \frac{1}{\eta\left(\frac{\tau+24 \lambda}{5}\right)} .
$$

If we also express the right-hand member of (1.1) in terms of $\eta(\tau)$, through its definition (1.3), we get

$$
\sum_{\lambda=0}^{4} \eta\left(\frac{\tau+24 \lambda}{5}\right)^{-1}=5^{2} \frac{\eta(5 \tau)^{5}}{\eta(\tau)^{6}}
$$

as a restatement of (1.1).

In a similar way (1.2) can be rewritten as

$$
\sum_{\lambda=0}^{6} \eta\left(\frac{\tau+24 \lambda}{7}\right)^{-1}=7^{2} \frac{\eta(7 \tau)^{3}}{\eta(\tau)^{4}}+7^{3} \frac{\eta(7 \tau)^{7}}{\eta(\tau)^{8}} .
$$

3. We are now going to subject (2.3) and (2.4) to modular transformations, of which we need to test only the generators

$$
S=\left(\begin{array}{ll}
1 & 1 \\
0 & 1
\end{array}\right), \quad T=\left(\begin{array}{rr}
0 & -1 \\
1 & 0
\end{array}\right) .
$$

The definition (1.3) shows that

$$
\eta(\tau+24)=\eta(\tau) .
$$

Consequently the range of the summation in the left-hand member of (2.3) can be replaced by "modulo 5." Therefore $S^{24}$ produces only a cyclical exchange of the terms of the sum and does not change the sum as a whole. It follows that $S$ and $S^{25}$ have the same effect on the left-hand member of (2.3), and since $S^{25}$ means the replacement of $(\tau+24 \lambda) / 5$ by $((\tau+24 \lambda) / 5)+5$, this effect is clearly the appearance of a multiplier $e^{-5 \pi i / 12}$ in each summand. On the other side, the substitution $S$, that is, $\tau \rightarrow \tau+1$, provides the multiplier

$$
e^{19 \pi i / 12}=e^{-5 \pi i / 12}
$$


also on the right-hand side. Thus the equation (2.3) goes over into itself under the substitution $S$.

Similarly we see that $S$ and $S^{49}$ have the same effect on the left-hand member of (2.4), viz., multiplication of each summand by

$$
e^{-7 \pi i / 12}
$$

This same factor is taken up also, as (1.3) shows, by each term of the righthand member of (2.4) so that the equation (2.4) also remains invariant under the substitution $S$.

4. This is not so with $T$. Through the substitution

$$
\tau \rightarrow-\tau^{-1}
$$

equation (2.3) goes over into

$$
\sum_{\lambda=0}^{4} \eta\left(\frac{-1+24 \lambda \tau}{5 \tau}\right)^{-1}=5^{2} \frac{\eta\left(-\frac{5}{\tau}\right)^{5}}{\eta\left(-\frac{1}{\tau}\right)^{6}}
$$

The left-hand member, which we designate by $L_{5}$, can be rewritten as

with

$$
L_{5}=\eta\left(\frac{-1}{5 \tau}\right)^{-1}+\sum_{\lambda=1}^{4} \eta\left(\frac{24 \lambda \frac{\tau+24 \lambda^{\prime}}{5}+b_{\lambda}}{5 \frac{\tau+24 \lambda^{\prime}}{5}-24 \lambda^{\prime}}\right)^{-1}
$$

$$
\lambda \lambda^{\prime} \equiv-1(\bmod 5)
$$

and

$$
b_{\lambda}=-\left(24^{2} \lambda \lambda^{\prime}+1\right) / 5 .
$$

Now for a modular substitution

we have [8],

$$
\left(\begin{array}{ll}
a & b \\
c & d
\end{array}\right), \quad c>0
$$

$$
\eta\left(\frac{a \tau+b}{c \tau+d}\right)=\exp \left(-\pi i\left(s(a, c)-\frac{a+d}{12 c}\right)\right)(-i(c \tau+d))^{1 / 2} \eta(\tau),
$$

where $s(a, c)$ is the "Dedekind sum"

$$
s(a, c)=\sum_{\mu \bmod c}\left(\left(\frac{\mu}{c}\right)\right)\left(\left(\frac{a \mu}{c}\right)\right)
$$


with

$$
((x))= \begin{cases}x-[x]-\frac{1}{2} & x \text { not an integer } \\ 0 & x \text { an integer. }\end{cases}
$$

If we apply (4.3) on (4.2) and the right-hand member of (4.1) for the modular substitutions

and

$$
\left(\begin{array}{rr}
0 & -1 \\
1 & 0
\end{array}\right)
$$

$$
\left(\begin{array}{cc}
24 \lambda & b_{\lambda} \\
5 & -24 \lambda^{\prime}
\end{array}\right)
$$

we obtain after a few reductions

$$
\frac{1}{5^{1 / 2} \eta(5 \tau)}+\sum_{\lambda=1}^{4} \frac{\exp \left(-\pi i\left(s(\lambda, 5)+\frac{2}{5}\left(\lambda-\lambda^{\prime}\right)\right)\right)}{\eta\left(\frac{\tau+24 \lambda^{\prime}}{5}\right)}=\frac{1}{5^{1 / 2}} \frac{\eta\left(\frac{\tau}{5}\right)^{5}}{\eta(\tau)^{6}}
$$

The Dedekind sums enjoy the following properties for

$$
\begin{aligned}
& (h, k)=1, h h^{\prime} \equiv-1(\bmod k): \\
& 12 k s(h, k) \equiv h-h^{\prime}(\bmod k), \\
& 12 k s(h, k) \equiv 0(\bmod 3),
\end{aligned}
$$

and, for $k$ odd,

$$
12 k s(h, k) \equiv k+1-2\left(\frac{h}{k}\right)(\bmod 8)
$$

with the Legendre-Jacobi symbol on the right-hand side of congruence (4.53) [9].

In our case $k=5,(\lambda, 5)=1$, we derive from these congruences

$$
\frac{1}{2} s(\lambda, 5)+\frac{\lambda-\lambda^{\prime}}{5} \equiv \frac{1}{4}\left(1+\left(\frac{\lambda}{5}\right)\right)(\bmod 1),
$$

and therefore

$$
\exp \left(-2 \pi i\left(\frac{1}{2} s(\lambda, 5)+\frac{1}{5}\left(\lambda-\lambda^{\prime}\right)\right)\right)=-\left(\frac{\lambda}{5}\right) .
$$

We remark that $\left(\lambda^{\prime} / 5\right)=(\lambda / 5)$ and obtain from (4.4) 


$$
5^{-1 / 2} \eta(5 \tau)^{-1}-\sum_{\lambda=1}^{4}\left(\frac{\lambda}{5}\right) \eta\left(\frac{\tau+24 \lambda}{5}\right)^{-1}=5^{-1 / 2} \frac{\eta\left(\frac{\tau}{5}\right)^{5}}{\eta(\tau)^{6}} .
$$

In virtue of (2.2) we can write for the sum on the left-hand side:

$$
\begin{aligned}
\sum_{\lambda=1}^{4} & =\sum_{\lambda=1}^{4}\left(\frac{\lambda}{5}\right) e^{-\pi i(\tau+24 \lambda) / 60} \sum_{n=0}^{\infty} p(n) e^{2 \pi i n(r+24 \lambda) / 5} \\
& =e^{-\pi i \tau / 60} \sum_{n=0}^{\infty} p(n) e^{2 \pi i n \tau / 5} \sum_{\lambda=1}^{4}\left(\frac{\lambda}{5}\right) e^{(2 \pi i \lambda / 5)(1-24 n)} \\
& =5^{1 / 2} e^{-\pi i \tau / 60} \sum_{n=0}^{\infty}\left(\frac{n+1}{5}\right) p(n) e^{2 \pi i n \tau / 5},
\end{aligned}
$$

where we have evaluated a Gaussian sum, and where for $5 \mid(n+1)$ the symbol $((n+1) / 5)$ means 0 , as customary. If we introduce this result in to (4.6), apply (2.2) to its first term and finally replace $e^{2 \pi i \tau / 5}$ by $x$ we obtain

$$
\sum_{n=0}^{\infty} p(n) x^{25 n}-5 \sum_{n=1}^{\infty}\left(\frac{n}{5}\right) p(n-1) x^{n}=\frac{\prod\left(1-x^{m}\right)^{5}}{\prod\left(1-x^{5 m}\right)^{6}},
$$

which is the new identity we wished to derive.

Incidentally, we can construct a formula which is free of infinite products. If we multiply (4.7) by (1.1) the right-hand side will appear as

$$
5 \frac{1}{\Pi\left(1-x^{m}\right)} \cdot \frac{1}{\Pi\left(1-x^{5 m}\right)} .
$$

These infinite products can be replaced by series by means of $(2.1)$, so that we get

$$
\begin{aligned}
\left\{\sum_{n=0}^{\infty} p(n) x^{25 n}-5 \sum_{n=1}^{\infty}\left(\frac{n}{5}\right) p(n-1) x^{n}\right\} \sum_{l=0}^{\infty} p(5 l+4) x^{l} & \\
& =5 \sum_{n=0}^{\infty} p(n) x^{n} \cdot \sum_{n=0}^{\infty} p(n) x^{5 n} .
\end{aligned}
$$

Comparison of coefficients would yield certain quadratic relations among the $p(n)$.

5. The identity (2.4) can be treated in the same manner. We give only a few highlights. Replacing $\tau$ by $-\tau^{-1}$ in (2.4) we have

$$
\sum_{\lambda=0}^{6} \eta\left(\frac{24 \lambda \tau-1}{7 \tau}\right)^{-1}=7^{2} \frac{\eta\left(-\frac{7}{\tau}\right)^{3}}{\eta\left(-\frac{1}{\tau}\right)^{4}}+7^{3} \frac{\eta\left(-\frac{7}{\tau}\right)^{7}}{\eta\left(-\frac{1}{\tau}\right)^{8}}
$$


Calling the left-hand member $L_{7}$ we rewrite it as

with

$$
L_{7}=\eta\left(-\frac{1}{7 \tau}\right)^{-1}+\sum_{\lambda=1}^{6} \eta\left(\frac{24 \lambda \frac{\tau+96 \lambda^{\prime}}{7}+b_{\lambda}}{7 \frac{\tau+96 \lambda^{\prime}}{7}-96 \lambda^{\prime}}\right)^{-1}
$$

$$
\lambda \lambda^{\prime} \equiv-1(\bmod 7)
$$

and

$$
b_{\lambda}=-\left(24 \cdot 96 \lambda \lambda^{\prime}+1\right) / 7 .
$$

By means of (4.3) the formula (5.2) goes over into

$$
L_{7}=(-7 i \tau)^{-1 / 2}+(-i \tau)^{-1 / 2} \sum_{\lambda=1}^{6} M_{\lambda \eta}\left(\frac{\tau+96 \lambda^{\prime}}{7}\right)^{-1},
$$

where we have

$$
M_{\lambda}=\exp \left\{\pi i\left(\left(s(24 \lambda, 7)-\frac{2 \lambda-8 \lambda^{\prime}}{7}\right)\right\} .\right.
$$

The congruences $(4.51),(4.52),(4.53)$ yield

$$
\frac{1}{2} s(24 \lambda, 7)-\frac{\lambda-4 \lambda^{\prime}}{7} \equiv \frac{1}{4}\left(\frac{\lambda}{7}\right)(\bmod 1),
$$

and therefore

$$
M_{\lambda}=\exp \left\{\frac{\pi i}{2}\left(\frac{\lambda}{7}\right)\right\}=i\left(\frac{\lambda}{7}\right)=-i\left(\frac{\lambda^{\prime}}{7}\right) .
$$

We introduce (5.2), (5.3), (5.4) into (5.1), carry out the modular transformation on the right-hand side, and get thereby

$$
\begin{aligned}
& 7^{-1 / 2} \eta(7 \tau)^{-1}-i \sum_{\lambda^{\prime}=1}^{6}\left(\frac{\lambda^{\prime}}{7}\right) \eta\left(\frac{\tau+96 \lambda^{\prime}}{7}\right)^{-1} \\
&=7^{1 / 2} \frac{\eta\left(\frac{\tau}{7}\right)^{3}}{\eta(\tau)^{4}}+7^{-1 / 2} \frac{\eta\left(\frac{\tau}{7}\right)^{7}}{\eta(\tau)^{8}} .
\end{aligned}
$$

The sum over $\lambda^{\prime}$ can be expanded into an infinite series by means of (2.2):

$$
\sum_{\lambda^{\prime}=1}^{6}\left(\frac{\lambda^{\prime}}{7}\right) \eta\left(\frac{\tau+96 \lambda^{\prime}}{7}\right)^{-1}=-i 7^{1 / 2} e^{-7 \pi i \tau / 12} \sum_{n=2}^{\infty}\left(\frac{n}{7}\right) p(n-2) e^{2 \pi i n \tau / 7}
$$


this derivation required the use of the Gaussian sum

$$
\sum_{\lambda^{\prime}=1}^{6}\left(\frac{\lambda^{\prime}}{7}\right) e^{2 \pi \lambda^{\prime}(96 n-4) / 7}=-i 7^{1 / 2}\left(\frac{n+2}{7}\right) .
$$

If we now insert (5.6) in (5.5), apply (2.2) and (1.3) in appropriate places, and finally change $e^{2 \pi i \tau / 7}$ into $x$ we obtain

$$
\begin{aligned}
\sum_{n=0}^{\infty} p(n) x^{49 n}-7 \sum_{n=2}^{\infty}\left(\frac{n}{7}\right) p(n & -2) x^{n} \\
& =7 x \frac{\prod\left(1-x^{m}\right)^{3}}{\prod\left(1-x^{7 m}\right)^{4}}+\frac{\Pi\left(1-x^{m}\right)^{7}}{\Pi\left(1-x^{7 m}\right)^{8}} .
\end{aligned}
$$

6. Up to this moment we have taken the Ramanujan identities (1.1) and (1.2) for granted and have inferred the identities (4.7) and (5.7) as direct consequences. From another point of view, however, we can take these new identities as bases for proofs of (1.1) and (1.2). For that purpose we consider the Ramanujan identities in the forms (2.3) and (2.4), which we prefer to write now as

$$
\sum_{\lambda=0}^{4} \eta(5 \tau) \eta\left(\frac{\tau+24 \lambda}{5}\right)^{-1}=25\left(\frac{\eta(5 \tau)}{\eta(\tau)}\right)^{6}
$$

and

$$
\sum_{\lambda=0}^{6} \eta(7 \tau) \eta\left(\frac{\tau+24 \lambda}{7}\right)^{-1}=7^{2}\left(\frac{\eta(7 \tau)}{\eta(\tau)}\right)^{4}+7^{3}\left(\frac{\eta(7 \tau)}{\eta(\tau)}\right)^{8} .
$$

We shall refer to these equations shortly in the abbreviations

$$
L_{5}^{*}(\tau)=R_{5}^{*}(\tau) ; \quad L_{7}^{*}(\tau)=R_{7}^{*}(\tau),
$$

respectively.

We can show that $L_{5}^{*}(\tau)$ and $R_{5}^{*}(\tau)$ are both modular functions of "level 5" ("stufe 5" in Felix Klein's terminology), that is, belonging to a congruence subgroup modulo 5 of the modular group. The subgroup in question is $\Gamma_{0}(5)$, characterized by $c \equiv 0(\bmod 5)$; it is of index 6 in the full modular group. As generators of $\Gamma_{0}(5)$ we can choose the substitutions (cf. [7, p. 147])

$$
S=\left(\begin{array}{ll}
1 & 1 \\
0 & 1
\end{array}\right), \quad V_{2}=\left(\begin{array}{rr}
-2 & -1 \\
5 & 2
\end{array}\right), \quad V_{3}=\left(\begin{array}{rr}
-3 & -1 \\
10 & 3
\end{array}\right)
$$

and need to test the invariance of $L_{5}^{*}(\tau)$ and $R_{5}^{*}(\tau)$ only with respect to these 3 substitutions. The discussion of $S$ has already essentially been done in $\$ 3$. The multiplier $e^{-5 \pi i / 12}$, which is mentioned there, is exactly absorbed by the factor $\eta(5 \tau)$ by which (6.1) differs from (2.3). 
As far as $R_{6}^{*}(\tau)$ is concerned we have, for $V_{2}$,

$$
\begin{aligned}
\eta\left(5 \frac{-2 \tau-1}{5 \tau+2}\right) & =\eta\left(\frac{-2 \cdot 5 \tau-5}{5 \tau+2}\right) \\
& =\exp \{-\pi i s(-2,1)\}(-i(5 \tau+2))^{1 / 2} \eta(5 \tau),
\end{aligned}
$$

in virtue of (4.3), and also

$$
\eta\left(\frac{-2 \tau-1}{5 \tau+2}\right)=\exp \{-\pi i s(-2,5)\}(-i(5 \tau+2))^{1 / 2} \eta(\tau) .
$$

Since $s(-2,1)=0$, as directly seen from (4.31) and (4.32), and

$$
s(-2,5)=-s(2,5)=0
$$

because of the property of the Dedekind sums

$$
s(h, k)=0
$$

for

$$
h^{2} \equiv-1(\bmod k)
$$

we have

$$
R_{5}^{*}\left(V_{2} \tau\right)=R_{5}^{*}(\tau)
$$

Similarly we find

$$
R_{5}^{*}\left(V_{3} \tau\right)=R_{5}^{*}(\tau) .
$$

As a matter of fact, not only $R_{b}^{*}(\tau)$ but already its sixth root $\eta(5 \tau) / \eta(\tau)$ is invariant with respect to $V_{2}$ and $V_{3}$, but not with respect to $S$.

The expression $L_{5}^{*}(\tau)$ goes over under $V_{2}$ into

$$
L_{5}^{*}\left(V_{2} \tau\right)=\sum_{\lambda=0}^{4} \eta\left(5 \frac{-2 \tau-1}{5 \tau+2}\right) \eta\left(\frac{1}{5}\left(\frac{-2 \tau-1}{5 \tau+2}+24 \lambda\right)\right)^{-1} .
$$

In order to bring this back into the previous form we need modular substitutions

$$
\left(\begin{array}{ll}
a & b \\
c & d
\end{array}\right)
$$

and a summation variable $\mu$ such that

$$
\frac{1}{5}\left(\frac{-2 \tau-1}{5 \tau+2}+24 \lambda\right)=\frac{a \frac{\tau+24 \mu}{5}+b}{c \frac{\tau+24 \mu}{5}+d} .
$$


A comparison of the coefficients of the linear functions of $\tau$ on both sides leads to

$$
\begin{array}{ll}
\mu=2-\lambda, \\
a=120 \lambda-2, & b=-24^{2} \lambda(2-\lambda)-19, \\
c=25, & d=-120(2-\lambda)+2 .
\end{array}
$$

We have therefore:

$$
L_{5}^{*}\left(V_{2} \tau\right)=\sum_{\lambda=0}^{4} \eta\left(\frac{-2 \cdot 5 \tau-5}{5 \tau+2}\right) \eta\left(\frac{a \frac{\tau+24(2-\lambda)}{5}+b}{c \frac{\tau+24(2-\lambda)}{5}+d}\right)^{-1},
$$

$a, b, c, d$ being taken from (6.3). Application of (4.3) now shows that

$$
L_{5}^{*}\left(V_{2} \tau\right)=\sum_{\lambda=0}^{4} M_{\lambda} \eta(5 \tau) \eta\left(\frac{\tau+24(2-\lambda)}{5}\right)^{-1}
$$

with the multiplier

$$
M_{\lambda}=\exp \left\{\pi i\left(s(120 \lambda-2,25)-\frac{240 \lambda-240}{300}\right)\right\} .
$$

Now we have

$$
s(120 \lambda-2,25)=-s(5 \lambda+2,25),
$$

and from (4.51), (4.52), (4.53)

$$
12 \cdot 25 s(5 \lambda+2,25) \equiv\left\{\begin{array}{l}
5 \lambda+2-(-5 \lambda+12)(\bmod 25) \\
0(\bmod 3) \\
25+1-2(\bmod 8)
\end{array}\right.
$$

from which we readily derive

$$
s(5 \lambda+2,25) \equiv-\frac{4}{5}(\lambda-1)(\bmod 2),
$$

so that

$$
M_{\lambda}=1 .
$$

Therefore

$$
L_{5}^{*}\left(V_{2} \tau\right)=L_{5}^{*}(\tau)
$$

is proved. Reasonings of a similar kind verify the equation

$$
L_{5}^{*}\left(V_{3} \tau\right)=L_{5}^{*}(\tau) .
$$

7. With $L_{5}^{*}(\tau)$ and $R_{5}^{*}(\tau)$ the difference 


$$
D_{5}(\tau)=L_{5}^{*}(\tau)-R_{5}^{*}(\tau)
$$

belongs also to $\Gamma_{0}(5)$. If we now can show that $D_{5}(\tau)$ remains bounded in the whole fundamental region it must be a constant. Now in the interior of the upper $\tau$-half-plane $\eta(\tau)$ is free of poles and zeros and $D_{5}(\tau)$ is therefore finite. The only parabolic points of the fundamental region of $\Gamma_{0}(5)$ are the points $\tau=i \infty$ and $\tau=0$. Now for $\tau \rightarrow i \infty$ it is readily seen that $D_{5}(\tau) \rightarrow 0$ since $L_{5}^{*}$ and $R_{5}^{*}$ tend separately to 0 , as their expansions in $e^{2 \pi i \tau}$, which can be taken from (1.3), show directly.

In order to test $D_{5}(\tau)$ for $\tau$ near 0 we carry out the substitution $\tau \rightarrow-\tau^{-1}$ and study $D_{5}\left(-\tau^{-1}\right)$ for $\tau$ near $i \infty$. This is now simple with (4.6), which in correspondence to (6.1) we shall have to write as

$$
5^{-1 / 2} \eta\left(\frac{\tau}{5}\right) \eta(5 \tau)^{-1}-\sum_{\lambda=1}^{4}\left(\frac{\lambda}{5}\right) \eta\left(\frac{\tau}{5}\right) \eta\left(\frac{\tau+24 \lambda}{5}\right)^{-1}
$$

$$
=5^{-1 j 2}\left(\frac{\eta\left(\frac{\tau}{5}\right)}{\eta(\tau)}\right)^{6} .
$$

Indeed, $D_{5}\left(-\tau^{-1}\right)$ is the difference of the two members of equation (7.1). In the uniformizing variable $e^{2 \pi i \tau / 5}$ both members have a pole of the first order at $i \infty$. If therefore the two sides of (7.1) agree in their first term the difference $D_{5}\left(-\tau^{-1}\right)$ remains bounded also at $i \infty$ or $D_{5}(\tau)$ at the second parabolic point $\tau=0$. Instead, however, of comparing the coefficients of the first term of the members of (7.1), it is easier to do it with (4.7). This is equivalent since (7.1) is obtained from (4.7) through the multiplication by

$$
5^{-1 / 2} x^{-1} \prod\left(1-x^{m}\right) \text {. }
$$

Now indeed both sides in (4.7) begin with the term 1 .

We have therefore proved that $D_{5}(\tau)$ is a constant, which can only be zero since $D_{5}(\tau) \rightarrow 0$ with $\tau \rightarrow i \infty$ as we have mentioned before. This proves (6.1) and therefore (2.3) and (1.1). Mordell in [2] also proves (6.1) by testing its two members at the parabolic points of $\Gamma_{0}(5)$. We used here for this purpose the independent theory of $\eta(\tau)$.

8. We can discuss (6.2) in the same manner. First we have to show that $L_{7}^{*}(\tau)$ and $R_{7}^{*}(\tau)$ are modular functions of level 7 , belonging to $\Gamma_{0}(7)$ with $c \equiv 0(\bmod 7)$. This step we could perform in analogy to the procedure in $\S 6$ by testing the generating substitutions of $\Gamma_{0}(7)$ which we can take as

$$
S=\left(\begin{array}{ll}
1 & 1 \\
0 & 1
\end{array}\right), \quad V_{3}=\left(\begin{array}{rr}
-2 & -1 \\
7 & 3
\end{array}\right), \quad V_{5}=\left(\begin{array}{rr}
-4 & -1 \\
21 & 5
\end{array}\right),
$$

(cf. [8]). Such a procedure, however, would not only mean a repetition of previous arguments but would involve a good deal of numerical work, for 
which, by the way, the congruences (4.51)-(4.53) would not quite suffice as a basis $\left({ }^{2}\right)$. We prefer therefore to discuss (6.2) on a more general ground, by taking recourse to the following theorems, in which $p$ always designates a prime number greater than 3 .

THEOREM 1. The functions

$$
\Phi_{p, r}(\tau)=\left(\frac{\eta(p \tau)}{\eta(\tau)}\right)^{r}
$$

with

$$
r(p-1) \equiv 0(\bmod 24)
$$

have the transformation equation

$$
\Phi_{p, r}(V \tau)=\left(\frac{a}{p}\right)^{r} \Phi_{p, r}(\tau)
$$

for the modular substitution

$$
V \tau=\frac{a \tau+b}{c \tau+d}
$$

of $\Gamma_{0}(p),(a / p)$ being the Legendre symbol.

Theorem 2. The function

$$
L_{p}^{*}(\tau)=\sum_{\lambda=0}^{p-1} \eta(p \tau) \eta\left(\frac{\tau+24 \lambda}{p}\right)^{-1}
$$

is invariant under the modular substitutions of $\Gamma_{0}(p)$ with $c \equiv 0(\bmod p)$.

In order not to interrupt the present line of thought we postpone the proof of these theorems to Part III of this paper.

For $p=7$ and $r=4$ and 8 the Theorems 1 and 2 show immediately that $D_{7}(\tau)=L_{7}^{*}(\tau)-R_{7}^{*}(\tau)$ as taken from (6.2) is an invariant of $\Gamma_{0}(7)$. We have now to show that $D_{7}(\tau)$-remains bounded in the fundamental region of this group. The only parabolic points of that region are again the points $\tau=i \infty$ and $\tau=0$. For $\tau \rightarrow i \infty$ we have $D_{7}(\tau) \rightarrow 0$, since $L_{7}^{*}(\tau)$ and $R_{7}^{*}(\tau)$ tend separately to 0 , in virtue of the factor $e^{\pi i \tau / 12}$ before the infinite product in the definition (1.3) for $\eta(\tau)$.

Instead of investigating $D_{7}(\tau)$ directly for $\tau \rightarrow 0$ we carry out the substitution $T \tau=-\tau^{-1}$ and then let $\tau$ tend to $i \infty$. But this substitution has been studied in $\S 5$. We have therefore $D_{7}\left(-\tau^{-1}\right)$ as the difference of the two members of the equation

(2) Cf. Lemmas 1 and 3, \$13. 


$$
\begin{aligned}
7^{-1 / 2} \eta\left(\frac{\tau}{7}\right) \eta(7 \tau)^{-1}-i \sum_{\lambda^{\prime}=1}^{6}\left(\frac{\lambda^{\prime}}{7}\right) \eta\left(\frac{\tau}{7}\right) \eta\left(\frac{\tau+96 \lambda^{\prime}}{7}\right)^{-1} & \\
& =7^{1 / 2}\left(\frac{\eta\left(\frac{\tau}{7}\right)}{\eta(\tau)}\right)^{4}+7^{-1 / 2}\left(\frac{\eta\left(\frac{\tau}{7}\right)}{\eta(\tau)}\right)^{8},
\end{aligned}
$$

which is obtained from (5.5) by multiplication with $\eta(\tau / 7)$ and which is the result of the transformation of (6.2). The application of (1.3) shows that each member of (8.5) begins with terms in $e^{-4 \pi i \tau / 7}$, or, in other words has a pole of the second order in the uniformizing variable $e^{2 \pi i \tau / 7}$. If we can therefore verify that the two members of (8.5) have their pole terms, the first two terms, in common, then the difference $D_{7}\left(-\tau^{-1}\right)$ remains bounded also at $\tau=i \infty$, and is bounded in the whole fundamental region. The comparison of the first two terms of each side of (8.5) is much easier to carry out in (5.7), which through multiplication by

$$
7^{-1 / 2} x^{-2} \prod\left(1-x^{m}\right)
$$

goes over into (8.5). Now the first two coefficients of both sides of (5.7) are indeed in agreement, they are 1 and 0 for both.

Since therefore $D_{7}(\tau)$ is bounded in the fundamental region it is a constant, and this constant is obviously 0 , since $D_{7}(\tau) \rightarrow 0$ for $\tau \rightarrow i \infty$, as mentioned. But $D_{7}(\tau)=0$ means that the equation (6.2) must hold, and this is equivalent to a proof of (1.2) (cf. [2]).

9. All these reasonings apply also to an identity which Zuckerman [4] has given in the form

$$
\sum_{l=0}^{\infty} p(13 l+6) x^{l}=\sum_{j=0}^{6} a_{j} x^{j} \frac{\prod\left(1-x^{13 m}\right)^{2 j+1}}{\prod\left(1-x^{m}\right)^{2(j+1)}},
$$

where the $a_{j}$ are certain integers which are computed in Zuckerman's paper. The procedure which we applied to (1.1) and (1.2) in $\$ \S 4$ and 5 leads here to the transformed identity

$$
\begin{aligned}
\sum_{n=0}^{\infty} p(n) x^{169 n}-13 \sum_{n=7}^{\infty}\left(\frac{n}{13}\right) p(n & -7) x^{n} \\
& =\sum_{j=0}^{6} a_{j} 13^{1-j} x^{6-j} \frac{\prod\left(1-x^{m}\right)^{2 j+1}}{\prod\left(1-x^{13 m}\right)^{2(j+1)}} .
\end{aligned}
$$

The numbers $13^{1-i} a_{j}$ are integers.

Our method yields now a direct proof of (9.1). We first express (9.1) and (9.2) in terms of $\eta(\tau)$. We have only to observe that $x=e^{2 \pi i \tau}$ in $(9.1)$ and $x=e^{2 \pi i \tau / 13}$ in (9.2). Moreover we multiply the resulting equations by $\eta(13 \tau)$ and $\eta(\tau / 13)$, respectively. 
For the proof of (9.1) we have first to show that

and

$$
\left(\frac{\eta(13 \tau)}{\eta(\tau)}\right)^{2}
$$

$$
\sum_{\lambda=0}^{12} \eta(13 \tau) \eta\left(\frac{\tau+24 \lambda}{13}\right)^{-1}
$$

belong to the group $\Gamma_{0}(13)$ with $c \equiv 0(\bmod 13)$. This is at once inferred from the Theorems 1 and 2 for $p=13$ and $r=2$.

Secondly, we simply have to compare the first 7 coefficients of (9.2) since $\eta(\tau / 13) \eta(13 \tau)^{-1}$ as well as $\eta(\tau / 13)^{14} \eta(\tau)^{-14}$ begin with $e^{-14 \pi i \tau / 13}$, that is, have a pole of 7 th order in the uniformizing variable $e^{2 \pi i \tau / 13}$. Now the comparison of the first seven coefficients of (9.2) yields without too much effort the following seven equations, with $b_{j}=13^{1-i} a_{j}$ :

$$
\begin{aligned}
& 1=b_{6}, \\
& 0=-13 b_{6}+b_{5} \\
& 0=65 b_{6}-11 b_{5}+b_{4}, \\
& 0=-130 b_{6}+44 b_{5}-9 b_{4}+b_{3}, \\
& 0=-65 b_{6}-55 b_{5}+9 b_{4}-7 b_{3}+b_{2}, \\
& 0=728 b_{6}-110 b_{5}-12 b_{4}+14 b_{3}-5 b_{2}+b_{1}, \\
& 0=-871 b_{6}+484 b_{5}-90 b_{4}+7 b_{3}+5 b_{2}-3 b_{1}+b_{0} .
\end{aligned}
$$

But these are exactly the equations by which Zuckerman (p. 104 of his paper) determines the coefficients which we here have called $a_{j}$. His derivation of these equations is based on an entirely different argument.

\section{Part II. Inentities of Watson and Zuckerman}

10. G. N. Watson [3] and H. S. Zuckerman [4] have derived identities analogous to those of Ramanujan, but corresponding to powers of 5 and 7 as moduli :

$$
\sum_{l=0}^{\infty} p(25 l+24) x^{l}=\sum_{j=1}^{5} b_{j} x^{j-1} \frac{\Pi\left(1-x^{5 m}\right)^{6 j}}{\prod\left(1-x^{m}\right)^{6 j+1}}
$$

and

$$
\sum_{l=0}^{\infty} p(49 l+47) x^{l}=\sum_{j=1}^{14} c_{j} x^{j-1} \frac{\Pi\left(1-x^{7 m}\right)^{4 j}}{\Pi\left(1-x^{m}\right)^{4 j+1}} .
$$

The $b_{j}$ and $c_{j}$ are integers, which are computed in [4] and which incidentally, in accordance with Ramanujan's theorems about $p(25 l+24)$ and $p(49 l+47)$. have the properties $25 \mid b_{j}$ and $49 \mid c_{j}$. From our present point of view we can 
easily obtain a proof for (10.1) and (10.2). It may be sufficient to carry it out only for the first of these equations.

If in (6.1) we replace $\tau$ by $(\tau+24 \mu) / 5$ we get

$$
\sum_{\lambda, \mu=0}^{4} \eta(\tau) \eta\left(\frac{\tau+24 \mu+5 \cdot 24 \lambda}{25}\right)^{-1}=5^{2} \sum_{\mu=0}^{4}\left(\frac{\eta(\tau)}{\eta\left(\frac{\tau+24 \mu}{5}\right)}\right)^{6}
$$

or

$$
\sum_{\lambda=0}^{24} \eta(\tau) \eta\left(\frac{\tau+24 \lambda}{25}\right)^{-1}=5^{2} \Psi_{5,6}(\tau)
$$

with

$$
\Psi_{5,6}(\tau)=\sum_{\mu=0}^{4}\left(\frac{\eta(\tau)}{\eta\left(\frac{\tau+24 \mu}{5}\right)}\right)^{6}
$$

Now $\Psi_{6,6}(\tau)$ belongs to the group $\Gamma_{0}(5)$ as we infer from the following theorem, whose proof we defer to Part III.

THEOREM 3. The functions

$$
\Psi_{p, r}(\tau)=\sum_{\lambda=0}^{p-1}\left(\frac{\eta(\tau)}{\eta\left(\frac{\tau+24 \lambda}{p}\right)}\right)^{r}
$$

with $r(p-1) \equiv 0(\bmod 24)$ have the transformation equation

$$
\Psi_{p, r}(V \tau)=\left(\frac{a}{p}\right)^{r} \Psi_{p, r}(\tau)
$$

for the substitution

$$
V \tau=\frac{a \tau+b}{c \tau+d}
$$

with $c \equiv 0(\bmod p), p$ being a prime greater than 3 .

We can therefore try to construct $\Psi_{5,6}(\tau)$ as a polynomial in ( $\left.{ }^{3}\right)$

$$
\Phi_{5,6}(\tau)=\left(\frac{\eta(5 \tau)}{\eta(\tau)}\right)^{6}
$$

(3) The background of this possibility is, of course, the fact that (1) $\Phi_{5,6}$ is univalent in the fundamental region, having only a zero of order one at $\tau=i \infty$ and (2) $\Psi_{6,6}$ as well as $\Phi_{6,6}$ are regular in the interior of the fundamental region. However, we do not need this remark, since the following arguments are self-sufficient. 
For this purpose we determine coefficients $\beta_{j}$ so that

$$
\Psi_{5,6}(\tau)=\sum_{j=1}^{N} \beta_{j} \Phi_{5,6 j}(\tau) .
$$

We need to verify this equation only at the two parabolic points of the fundamental region of $\Gamma_{0}(5)$, namely, at $\tau=i \infty$ and $\tau=0$. At the former point (10.5) is satisfied since both members tend to 0 as $\tau \rightarrow i \infty$. Instead of discussing (10.5) directly for $\tau=0$ we subject it first to the transformation $T$, which yields, by the device employed in (4.2),

$$
\begin{aligned}
\eta\left(-\frac{1}{\tau}\right)^{6} \eta\left(-\frac{1}{5 \tau}\right)^{-6}+\sum_{\lambda=1}^{4} \eta\left(-\frac{1}{\tau}\right)^{6} \eta\left(\frac{24 \lambda \frac{\tau+24 \lambda^{\prime}}{5}+b_{\lambda}}{5 \frac{\tau+24 \lambda^{\prime}}{5}-24 \lambda^{\prime}}\right)^{-6} \\
=\sum_{j=1}^{N} \beta_{j} \eta\left(-\frac{5}{\tau}\right)^{6 j} \eta\left(-\frac{1}{\tau}\right)^{-6 i}
\end{aligned}
$$

or, in analogy to (4.6),

$$
5^{-3}\left(\frac{\eta(\tau)}{\eta(5 \tau)}\right)^{6}+\sum_{\lambda=1}^{4}\left(\frac{\eta(\tau)}{\eta\left(\frac{\tau+24 \lambda}{5}\right)}\right)^{6}=\sum_{j=1}^{N} \beta_{j} 5^{-3 i}\left(\frac{\eta\left(\frac{\tau}{5}\right)}{\eta(\tau)}\right)^{6 j} .
$$

Here both members show poles at $\tau=i \infty$ : the left-hand member begins with a term in $e^{-2 \pi i \tau}$, whereas the right-hand member begins with a term in $e^{-2 \pi i r N / 5}$. We have therefore $N=5$, that is, a pole of order 5 in the uniformizing variable $e^{2 \pi i \tau / 5}$.

If now the coefficients $\beta_{i}, j=1, \cdots, 5$, are determined in such a way that both members of (10.6) agree in their pole terms at $\tau=i \infty$, that is, in the 5 first terms, then the difference of the members of (10.5) remains bounded throughout the fundamental region and is therefore a constant, which in particular must be equal to 0 . We rewrite (10.6) as

$$
\eta(5 \tau)^{-6}-5^{3} \eta\left(\frac{\tau}{5}\right)^{-6}+5^{3} \sum_{\lambda=0}^{4} \eta\left(\frac{\tau+24 \lambda}{5}\right)^{-6}=\sum_{j=1}^{5} \beta_{j} 5^{3(1-j)} \frac{\eta\left(\frac{\tau}{5}\right)^{6 j}}{\eta(\tau)^{6(j+1)}},
$$

which, if we introduce $e^{2 \pi i \tau / 5}=x$, and

$$
\frac{1}{\Pi\left(1-x^{m}\right)^{6}}=\sum_{n=0}^{\infty} p_{6}(n) x^{n}, \quad p_{6}(0)=1,
$$

leads to 


$$
\begin{aligned}
\sum_{n=0}^{\infty} p_{6}(n) x^{25 n}-5^{3} x^{6} \sum_{n=0}^{\infty} p_{6}(n) x^{n}+5^{4} x^{10} & \sum_{l=0}^{\infty} p_{6}(5 l+4) x^{l} \\
& =\sum_{j=1}^{5} \beta_{j} 5^{-3 j+3} x^{5-j} \frac{\prod\left(1-x^{m}\right)^{6 j}}{\prod\left(1-x^{5 m}\right)^{6(j+1)}} .
\end{aligned}
$$

We need to ensure only the agreement of the first 5 terms on each side, that is, the terms with $x^{0}, x, \cdots, x^{4}$. If we leave aside all unnecessary terms we have therefore to compute $\beta_{1}, \beta_{2}, \cdots, \beta_{5}$ from

$$
\sum_{j=1}^{5} \beta_{j} 5^{-3 j+3} x^{5-j} \prod_{m=1}^{4}\left(1-x^{m}\right)^{6 j}=1+O\left(x^{5}\right) .
$$

This is equivalent to 5 linear equations for $\beta_{j} 5^{-3 j+3}$, which are solved stepwise, beginning with $\beta_{5} 5^{-12}=1$. These, however, are exactly the equations which Zuckerman solves on pp. 100, 101 of his paper, and which we do not need to repeat here.

Therefore, the equation (10.5) is proved for $N=5$ and appropriate $\beta_{j}$, $j=1, \cdots, 5$. Comparing (10.5) with (10.31) we obtain

$$
\sum_{\lambda=0}^{24} \eta\left(\frac{\tau+24 \lambda}{25}\right)^{-1}=5^{2} \sum_{j=1}^{5} \beta_{j} \frac{\eta(5 \tau)^{6 j}}{\eta(\tau)^{6 j+1}} .
$$

This in turn is equivalent to (10.1) for $b_{j}=\beta_{j}$, which therefore is proved.

We remark that for the construction of a similar identity for the modulus $5^{3}$ the required step would be slightly different from that one described at the beginning of this paragraph. We should first have again to replace $\tau$ by $(\tau+24 \mu) / 5$ which would lead to

$$
\sum_{\lambda=0}^{124} \eta\left(\frac{\tau+24 \lambda}{125}\right)^{-1}=\sum_{j=1}^{5} \beta_{j} \sum_{\mu=0}^{4} \frac{\eta(\tau)^{6 j}}{\eta\left(\frac{\tau+24 \mu}{5}\right)^{6 j+1}} .
$$

In order to have modular functions belonging to $\Gamma_{0}(5)$ we should now have to multiply both sides by $\eta(5 \tau)$ (and not by $\eta(\tau)$ as in (10.31)). As a matter of fact, we could prove by theorems analogous to Theorems 1 to 3 that the functions

$$
\sum_{\mu=0}^{4} \frac{\eta(\tau)^{6 i} \eta(5 \tau)}{\eta\left(\frac{\tau+24 \mu}{5}\right)^{6 j+1}}
$$

and hence, in virtue of the preceding equation,

$$
\sum_{\lambda=0}^{124} \eta(5 \tau) \eta\left(\frac{\tau+24 \lambda}{125}\right)^{-1}
$$


belong to $\Gamma_{0}(5)$ and therefore admit of a representation as a polynomial in $\Phi_{5,6}(\tau)$. Watson [3], indeed, observes that in an induction from $5^{k}$ to $5^{k+1}$ two different kinds of procedures are required according to the parity of $k$.

We could discuss and prove (10.2) in a manner completely similar to that applied to (10.1). We refrain from giving the details since no new ideas are involved. It is, moreover, clear that our method proves the existence of similar identities for any power of 13 as modulus.

11. In the preceding paragraph we have applied the substitution $T$ to the right-hand side of (10.7) but not yet to its left-hand side. It is worth while to carry it out since it will lead to a modular equation. With $\tau^{\prime}=-\tau^{-1}$ we have

$$
\sum_{\lambda=0}^{24} \eta\left(\frac{\tau^{\prime}+24 \lambda}{25}\right)^{-1}=\sum_{\lambda=0}^{24} \eta\left(\frac{24 \lambda \tau-1}{25 \tau}\right)^{-1}
$$

$$
=\sum_{\mu=0}^{4} \eta\left(\frac{24 \mu \cdot 5 \tau-1}{5 \cdot 5 \tau}\right)^{-1}+\sum_{\lambda \bmod 25,(\lambda, 5)=1} \eta\left(\frac{24 \lambda \frac{\tau+24 \lambda^{\prime}}{25}+b_{\lambda}}{25 \frac{\tau+24 \lambda^{\prime}}{25}-24 \lambda^{\prime}}\right)^{-1}
$$

where $\lambda \lambda^{\prime} \equiv-1(\bmod 25)$ and

$$
b_{\lambda}=\frac{1}{25}\left(-24^{2} \lambda \lambda^{\prime}+1\right) .
$$

The first sum on the right side of (11.1) can be taken from (4.1), the second admits the application of (4.3), therefore

$$
\begin{aligned}
\sum_{\lambda=0}^{24} \eta\left(\frac{\tau^{\prime}+24 \lambda}{25}\right)^{-1}= & 5^{2} \frac{\eta\left(-\frac{1}{\tau}\right)^{5}}{\eta\left(-\frac{1}{5 \tau}\right)^{6}} \\
& +(-i \tau)^{-1 / 2} \sum_{\lambda \bmod 25,(\lambda, 5)=1} M_{\lambda \eta}\left(\frac{\tau+24 \lambda}{25}\right)^{-1}
\end{aligned}
$$

with

$$
M_{\lambda}=\exp \left\{-\pi i\left(s(24 \lambda, 25)-\frac{24 \lambda-24 \lambda^{\prime}}{12 \cdot 25}\right)\right\}=1
$$

in virtue of the congruences (4.51), (4.52), (4.53). If we apply the substitution $T$ also to the right-hand side of $(10.7)$ we obtain

$$
\frac{1}{5} \frac{\eta(\tau)^{5}}{\eta(5 \tau)}+\sum_{\lambda \bmod } \sum_{25,(\lambda, 5)=1} \eta\left(\frac{\tau+24 \lambda}{25}\right)^{-1}=5^{2} \sum_{j=1}^{5} \beta_{j} 5^{-3 j} \frac{\eta\left(\frac{\tau}{5}\right)^{6 j}}{\eta(\tau)^{6 j+1}} .
$$


We could now, following the procedure of $\S \S 4,5$, introduce here infinite series with $p(n)$ as coefficients and infinite products. Instead of doing that we write

$$
\sum_{\lambda \bmod 25,(\lambda, 5)=1} \eta\left(\frac{\tau+24 \lambda}{25}\right)^{-1}=\sum_{\lambda=0}^{24} \eta\left(\frac{\tau+24 \lambda}{25}\right)^{-1}-\sum_{\mu=0}^{4} \eta\left(\frac{\tau+24 \cdot 5 \mu}{25}\right)^{-1} .
$$

Here we apply (10.7) and (2.2), the latter with $\tau / 5$ instead of $\tau$, so that (11.2) goes over into

$$
\frac{1}{5} \frac{\eta(\tau)^{5}}{\eta(5 \tau)^{6}}+5^{2} \sum_{j=1}^{5} \beta_{j} \frac{\eta(5 \tau)^{6 j}}{\eta(\tau)^{6 j+1}}-5^{2} \frac{\eta(\tau)^{5}}{\eta\left(\frac{\tau}{5}\right)^{6}}=5^{2} \sum_{j=1}^{5} \beta_{j} 5^{-3 j} \frac{\eta\left(\frac{\tau}{5}\right)^{6 j}}{\eta(\tau)^{6 j+1}} .
$$

If we multiply by $5 \eta(\tau)$ and replace $\tau$ by $5 \tau$ we get

$$
\Phi(5 \tau)^{-1}-5^{3} \Phi(\tau)+5^{3} \sum_{j=1}^{5} \beta_{j}\left(\Phi(5 \tau)^{j}-5^{-3 j} \Phi(\tau)^{-\jmath}\right)=0
$$

with

$$
\Phi(\tau)=\Phi_{5,6}(\tau)=\left(\frac{\eta(5 \tau)}{\eta(\tau)}\right)^{6} .
$$

We have already found in $\S 6$, and again by means of Theorem 2 , that $\Phi(\tau)$ is a modular function of level 5 . Therefore (11.3) is a transformation equation of level 5 and order 5 . In the form (11.3) it is reducible. We put

$$
5^{-3} \Phi(\tau)^{-1}=X, \quad \Phi(5 \tau)=Y,
$$

and have then, after multiplication of (11.3) by $X Y$,

$$
X-Y+5^{3} X Y \sum_{j=1}^{5} \beta_{j}\left(Y^{j}-X^{j}\right)=0,
$$

and after the exclusion of the factor

$$
5^{3} X Y \sum_{j=1}^{5} \beta_{j} \sum_{v=0}^{j-1} X^{v} Y^{j-1-v}=1 .
$$

This equation is of degree 5 in $Y$, as it has to be, because $\Phi(5 \tau)$ belongs to the group $\Gamma_{0}(25)$ with $c \equiv 0(\bmod 25)$, which is of index 5 in the group $\Gamma_{0}(5)$ of $\Phi(\tau)$. Therefore (11.5) is irreducible.

Moreover the equation (11.5) is symmetric in $X$ and $Y$. These two functions go over into each other by the substitution

$$
\tau^{\prime}=-(5 \tau)^{-1}
$$

With these properties the equation (11.5) fulfills the definition of a modular 
equation (Klein-Fricke $[5$, vol. 2 , pp. 56,57$]$ ), it is a modular equation of level 5 and order 5.

There exists also an equation for

$$
\frac{\eta(5 \tau)}{\eta(\tau)}=(\Phi(\tau))^{1 / 6}
$$

which in our notation is

(11.7) $5^{5 / 2} X^{5 / 6} Y^{1 / 6}+5^{3} X^{4 / 6} Y^{2 / 6}+3 \cdot 5^{5 / 2} X^{3 / 6} Y^{3 / 6}+5^{3} X^{2 / 6} Y^{4 / 6}+5^{5 / 2} X^{1 / 6} Y^{5 / 6}=1$

(cf. $[6$, p. 395 , formula (23)], and $[3$, p. 105, formula (3.2)]). The function (11.6) does not belong to $\Gamma_{0}(5)$, it is in fact a function of level 30 . We can look upon (11.7) from our present point of view as on a modular equation of level 5 in "irrational form." By elementary algebraic processes the equation (11.5) can be regained from (11.7).

12. A similar treatment of (10.2) leads to an algebraically different situation. Instead of (11.3) we obtain this time

$$
\begin{aligned}
7\left(\Phi(7 \tau)^{-1}-7^{2} \Phi(\tau)\right)+\left(\Phi(7 \tau)^{-2}-\right. & \left.7^{4} \Phi^{2}(\tau)\right) \\
& +7^{3} \sum_{j=1}^{14} c_{j}\left(\Phi(7 \tau)^{j}-7^{-2 j} \Phi(\tau)^{-j}\right)=0
\end{aligned}
$$

with

$$
\Phi(\tau)=\Phi_{7,4}(\tau)=\left(\frac{\eta(7 \tau)}{\eta(\tau)}\right)^{4} .
$$

Theorem 2 shows that $\Phi(\tau)$ is a modular function of level 7 , belonging to $\Gamma_{0}(7)$ and (12.11) is therefore a transformation equation of level 7 and order 7 . Let us put

$$
7^{-2} \Phi(\tau)^{-1}=X, \quad \Phi(7 \tau)=Y,
$$

so that (12.11) can be written as

$$
\left(Y^{-2}-X^{-2}\right)+7\left(Y^{-1}-X^{-1}\right)+7^{3} \sum_{j=1}^{14} c_{j}\left(Y^{j}-X^{j}\right)=0 .
$$

This equation can be freed of the factor $Y-X$. But then it would still remain of degree 15 in $Y$, whereas $\Phi(7 \tau)$ belongs to $\Gamma_{0}(49)$ which is of index 7 in $\Gamma_{0}(7)$. Therefore even after division by $Y-X$ equation (12.2) cannot be irreducible. If we write it indeed in the form

$$
Y^{-2}+7 Y^{-1}+7^{3} \sum_{j=1}^{14} c_{j} Y^{j}+C=X^{-2}+7 X^{-1}+7^{3} \sum_{j=1}^{14} c_{j} X^{j}+C
$$

it turns out by actual computation that for $C=(1 / 4) 7^{2} \cdot 32145$ and the $c_{j}$ which are numerically given in Zuckerman's paper [4] we have 


$$
\begin{aligned}
X^{-2}+7 X^{-1}+C+7^{3} \sum_{j=1}^{14} & c_{j} X^{j} \\
= & \left(X^{-1}+7 / 2+82 \cdot 7^{4} X+176 \cdot 7^{6} X^{2}+845 \cdot 7^{7} X^{3}\right. \\
& \left.+272 \cdot 7^{9} X^{4}+46 \cdot 7^{11} X^{5}+4 \cdot 7^{13} X^{6}+7^{14} X^{7}\right)^{2}
\end{aligned}
$$

a complete square. This permits the extraction of the square root in (12.3). The square root must be taken with the same sign on both sides so that the constant term disappears and a further division by $X-Y$ can reach the degree 7 of the equation which before that division is

$$
\begin{aligned}
\left(Y^{-1}-X^{-1}\right) & +82 \cdot 7^{4}(Y-X)+176 \cdot 7^{6}\left(Y^{2}-X^{2}\right)+845 \cdot 7^{7}\left(Y^{3}-X^{3}\right) \\
& +272 \cdot 7^{9}\left(Y^{4}-X^{4}\right)+46 \cdot 7^{11}\left(Y^{5}-X^{5}\right)+4 \cdot 7^{13}\left(Y^{6}-X^{6}\right) \\
& +7^{14}\left(Y^{7}-X^{7}\right)=0 .
\end{aligned}
$$

Multiplication by $X Y$ and then division by $Y-X$ establish an equation which is symmetric in $X$ and $Y$ and of degree 7 in $Y$ and therefore irreducible, since 7 is the index of the group $Y$ in that of $X$, as mentioned above. This equation is therefore a modular equation of level 7 and order 7. Again, an "irrational form" of it is known for

$$
\frac{\eta(7 \tau)}{\eta(\tau)}=(\Phi(\tau))^{1 / 4}
$$

It was given by Watson $[3$, p. 118, (5.2)] and appears in our notation as

$$
\begin{aligned}
7^{7 / 2} X^{7 / 4} Y^{1 / 4} & +7^{4} X^{6 / 4} Y^{2 / 4}+3 \cdot 7^{7 / 2} X^{5 / 4} Y^{3 / 4} \\
& +7^{4} X^{4 / 4} Y^{4 / 4}+3 \cdot 7^{7 / 2} X^{3 / 4} Y^{5 / 4}+7^{4} X^{2 / 4} Y^{6 / 4}+7^{7 / 2} X^{1 / 4} Y^{7 / 4} \\
& +7^{5 / 2} X^{3 / 4} Y^{1 / 4}+5 \cdot 7^{2} X^{2 / 4} Y^{2 / 4}+7^{5 / 2} X^{1 / 4} Y^{3 / 4}=1 .
\end{aligned}
$$

The equation (12.5) has as roots the 4 th powers of the roots of (12.7) and can therefore (disregarding the factor $Y-X$ in (12.5)) be directly derived from (12.6). A control by numerical calculation shows indeed complete agreement in the coefficients. The function (12.6), by the way, is of level 28 .

\section{Part III. Proofs for the Theorems 1, 2, and 3}

13. The proofs will be based on three lemmas, which occur as Theorems $17,18,19$ in [9].

Lemma 1. Let $\Theta=\Theta_{k}$ denote 1 for $3 \nmid k$ and 3 for $3 \mid k$ so that $\Theta \cdot k$ is either prime to 3 or divisible by $3^{2}$. For $(h, k)=1$ we have

$$
12 h k s(h, k) \equiv h^{2}+1(\bmod \Theta \cdot k) \text {. }
$$

Moreover

$$
12 k s(h, k) \equiv 0(\bmod 3),
$$

if $3 \nmid k$. 
Lemma 2. For odd $k$ we have

$$
12 k s(h, k) \equiv k+1-2\left(\frac{h}{k}\right)(\bmod 8),
$$

where $(h / k)$ denotes the Legendre-Jacobi symbol.

LEMмA 3. If $k$ is equal to $2^{\lambda} l, \lambda \geqq 0$ and $l$ and $h$ are odd integers, then

$$
12 h k s(h, k) \equiv h^{2}+k^{2}+3 k+1+2 k\left(\frac{k}{h}\right)\left(\bmod 2^{\lambda+3}\right) .
$$

We derive first two further lemmas about the "Dedekind sums" $s(h, k)$. In the sequel $p$ will always be a prime number greater than 3 , and $r$ is an integer such that

$$
r(p-1) \equiv 0(\bmod 24) .
$$

The condition imposed on $p$ will be used in the form

$$
p^{2} \equiv 1(\bmod 24)
$$

Lemma 4. Let $a, b, c, d$ be integers with $a d-b c=1, c>0, p \mid c$. Put $c=p \cdot c_{1}$ and

$$
G=\left(s(a, c)-\frac{a+d}{12 c}\right)-\left(s\left(a, c_{1}\right)-\frac{a+d}{12 c_{1}}\right) .
$$

Then with $r$ satisfying (13.51) we have

$$
r G \equiv \frac{1}{2}\left\{1-\left(\frac{a}{p}\right)^{r}\right\}(\bmod 2) .
$$

Proof. From (13.1) we obtain

$$
12 a c\left(s(a, c)-\frac{a+d}{12 c}\right) \equiv a^{2}+1-a(a+d) \equiv-b c(\bmod \Theta c),
$$

where $\Theta=\Theta_{c}$ is defined as in Lemma 1. If we then apply (13.1) with $k=c / p=c_{1}$, we have, after multiplication by $p$,

$$
12 a c\left(s\left(a, c_{1}\right)-\frac{a+d}{12 c_{1}}\right) \equiv p a^{2}+p-p a(a+d) \equiv-p b c(\bmod \Theta c) .
$$

It has to be remarked that $\Theta$ has the same value in both congruences, since $c$ and $c_{1}$ have either both the factor 3 or not. With the definition (13.61) we obtain

$$
12 a c r G \equiv r(p-1) b c \equiv 0(\bmod \Theta c)
$$


For $3 \nmid c$ we have $12 c r G \equiv 0(\bmod 3)$ as direct consequence from (13.2) and (13.51). Therefore

$$
12 c r G \equiv 0(\bmod 3 c) .
$$

Now let $c$ be odd. Then Lemma 2 is applicable and yields for $k=c$

$$
12 c\left(s(a, c)-\frac{a+d}{12 c}\right) \equiv c+1-2\left(\frac{a}{c}\right)-(a+d)(\bmod 8),
$$

and for $k=c / p=c_{1}$

$$
12 c\left(s\left(a, c_{1}\right)-\frac{a+d}{12 c_{1}}\right) \equiv c+p-2 p\left(\frac{a}{c_{1}}\right)-p(a+d)(\bmod 8) .
$$

We can replace $\left(a / c_{1}\right)$ by $\left(a / c_{1}\right)\left(a / p^{2}\right)=(a / c p)=(a / c)(a / p)$, and get therefore

$$
\begin{aligned}
12 c r G & \equiv r(1-p)-2 r\left(\frac{a}{c}\right)\left\{1-p\left(\frac{a}{p}\right)\right\}+r(p-1)(a+d) \\
& \equiv \pm 2 r\left\{1-p\left(\frac{a}{p}\right)\right\}(\bmod 8),
\end{aligned}
$$

which implies

$$
12 c r G \equiv 0(\bmod 8)
$$

for $r$ even. For $r$ odd the condition $(13.51)$ necessitates $p \equiv 1(\bmod 8)$ and therefore

$$
1-p\left(\frac{a}{p}\right) \equiv 1-\left(\frac{a}{p}\right)(\bmod 4),
$$

so that $(a / p)=1$ leads also to (13.72). For $r$ odd together with $(a / p)=-1$ we find, however,

$$
12 c r G \equiv 4(\bmod 8)
$$

or

$$
12 c r G \equiv 12 c(\bmod 8) \text {. }
$$

The congruences (13.71), (13.72), (13.73) prove (13.62) for odd $c$.

For even $c=2^{\lambda} \gamma$ with $\gamma$ odd we make use of Lemma 3 and obtain for $k=c$ and $k=c / p=c_{1}$

$$
\begin{aligned}
12 a c\left(s(a, c)-\frac{a+d}{12 c}\right) & \equiv a^{2}+c^{2}+3 c+1+2 c\left(\frac{c}{a}\right)-a(a+d) \\
& \equiv c^{2}+3 c-b c+2 c\left(\frac{c}{a}\right)\left(\bmod 2^{\lambda+3}\right),
\end{aligned}
$$


and

and hence

$$
\begin{aligned}
12 a c\left(s\left(a, c_{1}\right)-\frac{a+d}{12 c_{1}}\right) & \equiv p a^{2}+\frac{c^{2}}{p}+3 c+p+2 c\left(\frac{c_{1}}{a}\right)-p a(a+d) \\
& \equiv \frac{c^{2}}{p}+3 c-p b c+2 c\left(\frac{c}{a}\right)\left(\frac{p}{a}\right)\left(\bmod 2^{\lambda+3}\right),
\end{aligned}
$$

$$
\begin{aligned}
12 a c r G & \equiv r \frac{c^{2}}{p}(p-1)+r(p-1) b c+2 r c\left(\frac{c}{a}\right)\left\{1-\left(\frac{p}{a}\right)\right\} \\
& \equiv \pm 2^{\lambda+1} r\left\{1-\left(\frac{p}{a}\right)\right\}\left(\bmod 2^{\lambda+3}\right),
\end{aligned}
$$

and therefore

$$
12 c r G \equiv 0\left(\bmod 2^{\lambda+3}\right)
$$

for $r$ even, as well as for $(p / a)=1$, which for the case of odd $r$ and thus $p \equiv 1(\bmod 4)$ is equivalent to $(a / p)=1$.

For $r$ odd in conjunction with $(a / p)=-1$ we have

$$
12 c r G \equiv 2^{\lambda+2}\left(\bmod 2^{\lambda+3}\right) .
$$

The congruences (13.71), (13.74), (13.75) establish (13.62) also for the case of an even $c=2^{\lambda} \gamma$. This finishes the proof of Lemma 4 .

Lemma 5. Let $a, b, c, d$ be integers with $a d-b c=1, c>0, p^{2} \mid c$. Then, with $c=p^{2} c_{2}$, we have

$$
H=\left(s(a, c)-\frac{a+d}{12 c}\right)-\left(s\left(a, c_{2}\right)-\frac{a+d}{12 c_{2}}\right) \equiv 0(\bmod 2) .
$$

Proof. For $k=c$ Lemma 1. yields

$$
12 a c\left(s(a, c)-\frac{a+d}{12 c}\right) \equiv a^{2}+1-a(a+d) \equiv-b c(\bmod \Theta c),
$$

and for $k=c / p^{2}=c_{2}$

$$
12 a c\left(s\left(a, c_{2}\right)-\frac{a+d}{c_{2}}\right) \equiv p^{2}\left(a^{2}+1\right)-p^{2} a(a+d) \equiv-p^{2} b c(\bmod \Theta c) .
$$

Therefore

$$
12 c H \equiv 0(\bmod \Theta c) .
$$

This congruence holds, modulo $3 c$, also if $3 \nmid c$ since then according to (13.2) 
$12 c s(a, c)$ and $12 c s\left(a, c_{2}\right)$ are separately divisible by 3 , and so is $\left(p^{2}-1\right) a(a+d)$. Thus we obtain

$$
12 c H \equiv 0(\bmod 3 c) .
$$

Now suppose, first, $c$ to be odd. Then we infer from Lemma 2

$$
12 c\left(s(a, c)-\frac{a+d}{12 c}\right) \equiv c+1+2\left(\frac{a}{c}\right)-(a+d)(\bmod 8),
$$

and

$$
12 c\left(s\left(a, c_{2}\right)-\frac{a+d}{12 c_{2}}\right) \equiv c+p^{2}+2 p^{2}\left(\frac{a}{c_{2}}\right)-p^{2}(a+d)(\bmod 8) .
$$

But since $\left(a / c_{2}\right)=(a / c)$ and $p^{2}-1 \equiv 0(\bmod 8)$ we find by subtraction

$$
12 c H \equiv 0(\bmod 8),
$$

which together with (13.91) proves (13.8) for odd $c$.

Secondly, in case we have $c=2^{\lambda} \gamma, \gamma$ and $a$ odd, we obtain from Lemma 3

and

$$
\begin{aligned}
12 a c\left(s(a, c)-\frac{a+d}{12 c}\right) & \equiv a^{2}+c^{2}+3 c+1+2 c\left(\frac{c}{a}\right)-a(a+d) \\
& \equiv c^{2}+3 c-b c+2 c\left(\frac{c}{a}\right)\left(\bmod 2^{\lambda+3}\right),
\end{aligned}
$$

and therefore

$$
\begin{aligned}
12 a c\left(s\left(a, c_{2}\right)-\frac{a+d}{12 c_{2}}\right) & \equiv p^{2} a^{2}+\frac{c^{2}}{p^{2}}+3 c+p^{2}+2 c\left(\frac{c_{2}}{a}\right)-p^{2} a(a+d) \\
& \equiv \frac{c^{2}}{p^{2}}+3 c-p^{2} b c+2 c\left(\frac{c}{a}\right)\left(\bmod 2^{\lambda+3}\right),
\end{aligned}
$$

$$
12 a c H \equiv \frac{c^{2}}{p^{2}}\left(p^{2}-1\right)+\left(p^{2}-1\right) b c \equiv 0\left(\bmod 2^{\lambda+3}\right),
$$

or

$$
12 c H \equiv 0\left(\bmod 2^{\lambda+3}\right) .
$$

This together with (13.91) completes the proof of (13.8) for $c$ even.

14. Proof of Theorem 1. Here and in the following proof we treat the modular substitutions with $c=0$ separately. Since all of these are iterations of $S \tau=\tau+1$, it suffices in this case to study only $S$. Now the definitions (8.1) and (1.3) show immediately

$$
\Phi_{p, r}(\tau+1)=e^{\pi i r(p-1) / 12} \Phi_{p, r}(\tau)
$$


which because of (8.2) amounts to

$$
\Phi_{p, r}(\tau+1)=\Phi_{p, r}(\tau) .
$$

This can be subsumed under (8.3) for $a=1$. From now on we can assume that $c>0$, with $c \equiv 0(\bmod p)$. The formula $(4.3)$ yields here

$$
\Phi_{p, r}(V \tau)=M_{V} \cdot \Phi_{p, r}(\tau)
$$

with

$$
M_{V}=\exp \left\{\pi i r\left(s(a, c)-\frac{a+d}{12 c}-s\left(a, c_{1}\right)+\frac{a+d}{12 c_{1}}\right)\right\}, \quad c_{1}=\frac{c}{p} .
$$

Application of Lemma 4 gives

$$
M_{V}=\exp \left\{\frac{\pi i}{2}\left(1-\left(\frac{a}{p}\right)^{r}\right)\right\} .
$$

This can be written briefly as

$$
M_{V}=\left(\frac{a}{p}\right)^{r}
$$

which proves Theorem 1 .

15. Proof of Theorem 2. We first consider the effect of $S$ on $L_{p}^{*}(\tau)$. The definitions (8.4) and (1.3) show that in the sum defining $L_{p}^{*}(\tau)$ it is only essential that $\lambda$ runs through a complete residue system modulo $p$. We can therefore write

$$
L_{p}^{*}(\tau)=\sum_{\lambda=0}^{p-1} \eta(p \tau) \eta\left(\frac{\tau+24 N+24 \lambda}{p}\right)^{-1}
$$

for any integer $N$. Hence we get

$$
L_{p}^{*}(\tau+1)=\sum_{\lambda=0}^{p-1} \eta(p(\tau+1)) \eta\left(\frac{\tau+24 N+1+24 \lambda}{p}\right)^{-1} .
$$

If we choose here

$$
24 N+1=p^{2}
$$

we obtain

$$
L_{p}^{*}(\tau+1)=\sum_{\lambda=0}^{p-1} \eta(p \tau+p) \eta\left(\frac{\tau+24 \lambda}{p}+p\right)^{-1}
$$

which is equal to $L_{p}^{*}(\tau)$ in virtue of (1.3). Having thus disposed of the case $c=0$ we can suppose from now on $c>0$ with $c \equiv 0(\bmod p)$. 
In

$$
V=\left(\begin{array}{ll}
a & b \\
c & d
\end{array}\right)
$$

we have therefore necessarily $a \not \equiv 0(\bmod p)$. Since only a complete residue system of the index of summation was essential we can also write

$$
L_{p}^{*}(\tau)=\sum_{\lambda=0}^{p-1} \eta(p \tau) \cdot \eta\left(\frac{\tau+\left(p^{2}-1\right) a \lambda}{p}\right)^{-1} .
$$

We obtain therefore, with $c=p c_{1}$,

$$
L_{p}^{*}(V \tau)=\sum_{\lambda=0}^{p-1} \eta\left(\frac{a p \tau+p b}{c_{1} p \tau+d}\right) \cdot \eta\left(\frac{1}{p}\left(\frac{a \tau+b}{c \tau+d}+\left(p^{2}-1\right) a \lambda\right)\right)^{-1} .
$$

Proceeding here as we did in $\$ 6$ for $p=5$ we wish to construct modular substitutions

such that

$$
\left(\begin{array}{ll}
A & B \\
C & D
\end{array}\right)
$$

$$
\frac{1}{p}\left(\frac{a \tau+b}{c \tau+d}+\left(p^{2}-1\right) a \lambda\right)=\frac{A\left(\tau+\left(p^{2}-1\right) d \mu\right) / p+B}{C\left(\tau+\left(p^{2}-1\right) d \mu\right) / p+D},
$$

where $A, B, C, D$ and $\mu$ will depend on $\lambda$.

Comparison of coefficients shows that the equations

$$
\begin{aligned}
a+c\left(p^{2}-1\right) a \lambda & =A, & b+d\left(p^{2}-1\right) a \lambda & =p B+A\left(p^{2}-1\right) d \mu, \\
p c & =C, & p d & =p D+C\left(p^{2}-1\right) d \mu
\end{aligned}
$$

are necessary and sufficient. It is clear that for any choice of $\lambda$ and $\mu$ the numbers $A, B, C, D$ are uniquely determined through (15.3) and satisfy

$$
\left|\begin{array}{ll}
A & B \\
C & D
\end{array}\right|=1 \text {. }
$$

We can now tie $\mu$ to $\lambda$ in such a way that $B$ will become an integer, whereas $A, C, D$ are obviously integers for any integers $\lambda, \mu$. Since $p$ divides $c$ we have

$$
a \equiv A(\bmod p)
$$

and therefore

$$
b-a d \lambda \equiv-a d \mu(\bmod p),
$$

which implies already that $B$ is an integer. Now

and therefore

$$
1=a d-b c \equiv a d(\bmod p) \text {, }
$$




$$
\mu \equiv \lambda-b(\bmod p) .
$$

The new summation index $\mu$ needs only to be determined modulo $p$ and we can therefore, without loss of generality, put

$$
\mu=\lambda-b .
$$

This choice now completes the determination of $A, B, C, D$, which become

$$
\begin{aligned}
A & =a+c\left(p^{2}-1\right) a \lambda, & B & =p a d b-\frac{c}{p} b^{2}-\frac{c}{p}\left(p^{2}-1\right)^{2} a d \lambda(\lambda-b), \\
C & =p c, & D & =d-c\left(p^{2}-1\right) d(\lambda-b) .
\end{aligned}
$$

From (15.1), (15.2), and (4.3) we derive now

$$
L_{p}^{*}(V \tau)=\sum_{\lambda=0}^{p-1} M_{\lambda} \cdot \eta(p \tau) \cdot \eta\left(\frac{\tau+\left(p^{2}-1\right) d \mu}{p}\right)^{-1}
$$

with

$$
M_{\lambda}=\exp \left\{-\pi i\left(\left(s\left(a, c_{1}\right)-\frac{a+d}{12 c_{1}}\right)-\left(s(A, C)-\frac{A+D}{12 C}\right)\right)\right\} .
$$

From (15.6) it follows that

$$
a \equiv A(\bmod c)
$$

This permits us to write

$$
s\left(a, c_{1}\right)=s\left(A, c_{1}\right)=s\left(A, C_{2}\right), \quad C_{2}=\frac{C}{p^{2}}=\frac{c}{p}=c_{1} .
$$

Moreover the equations (15.6) show that $(a+d) / 12 c$, and $(A+D) / 12 c$ differ only by an even integer. We can therefore write

$$
M_{\lambda}=\exp \left\{-\pi i\left(\left(s\left(A, C_{2}\right)-\frac{A+D}{12 C_{2}}\right)-\left(s(A, C)-\frac{A+D}{12 C}\right)\right)\right\},
$$

and obtain then from Lemma 4

$$
M_{\lambda}=1 \text {. }
$$

If we observe that in (15.7) $\mu$ runs with $\lambda$ through a complete residue system modulo $p$ we have proved

$$
L_{p}^{*}(V \tau)=L_{p}^{*}(\tau),
$$

which finishes the proof of Theorem 2.

16. Proof of Theorem 3. The proof is closely similar to that of Theorem 2. The same auxiliary substitutions are used. It is then only necessary to apply Lemma 4 instead of Lemma 5 for the computation of the multiplier $M_{\lambda}$. 


\section{REFERENCES}

1. S. Ramanujan, Some properties of $p(n)$, the number of partitions of $n$, Collected Papers, pp. 210-213.

2. L. J. Mordell, Note on certain modular relations considered by Messrs. Ramanujan, Darling, and Rogers, Proceedings of the London Mathematical Society, (2), vol. 20 (1922), pp. $408-416$.

3. G. N. Watson, Ramanujans Vermutung über Zerfällungsanzahlen, Journal für die reine und angewandte Mathematik, vol. 179 (1938), pp. 97-128.

4. H. S. Zuckerman, Identities analogous to Ramanujan's identities involving the partition function, Duke Mathematical Journal, vol. 5 (1939), pp. 88-110.

5. F. Klein und R. Fricke, Vorlesungen über die Theorie der Elliptischen Modulfunktionen, vol. 1, 1890, and vol. 2, 1892.

6. R. Fricke, Die Elliptischen Funktionen und ihre Anwendungen, vol. 1, 1916, and vol. 2, 1922.

7. H. Rademacher, Über die Erzeugenden von Kongruenzuntergruppen der Modulgruppe, Abhandlungen aus dem Mathematischen Seminar der Hamburgischen Universität, vol. 7 (1930), pp. 134-148.

8. — Zur Theorie der Modulfunktionen, Journal für die reine und angewandte Mathematik, vol. 167 (1931), pp. 312-336.

9. H. Rademacher and A. Whiteman, On Dedekind sums, American Journal of Mathematics, vol. 63 (1941), pp. 377-407.

University of Pennsylvania, Philadelphia, Pa. 